SLAC-PUB-8323

December 1999

\title{
Assessment and Outlook*
}

\author{
Burton Richter \\ Stanford Linear Accelerator Center \\ Stanford, California 94309
}

Concluding Remarks presented at the

XIX International Symposium on Lepton and Photon Interactions at High Energies Stanford University, August 9-14, 1999

*Work supported by the Department of Energy under contract number DE-AC03-76SF00515. 


\section{Introduction}

My assignment at this conference is to assess where we are in high-energy physics and speculate on where we might be going. This frees me from any obligation to summarize all that went on here and allows me to talk just about those topics that interest me the most at this time. I will let my prejudices show and talk a bit about physics in general, CP violation, neutrinos, accelerators, non-accelerator experiments, and even theory.

I start with a bit of my own history in physics and what I see as cycles in science-I think these are relevant. I began research in physics when I was in my third undergraduate year at MIT. I worked with Professor Francis Bitter in his magnet laboratory and learned how to do an experiment. I spent three years with Francis Bitter, but, as I began my second year of graduate school, I found myself becoming less interested in the nuclear magnetic moments that I was trying to measure and more interested in the fundamentals of the protons and neutrons that contributed to the moments. I shifted to particle physics, never regretting it.

When in graduate school I did both theory and experiment. It was not hard to do that back then because, if you could manipulate $\gamma$ matrices, set up and solve integral equations and understood what a Green's function was, you could keep up with theory and even do some of your own. I think that an experimenter in graduate school today would not have an easy time keeping up with the sophisticated mathematics required to understand what's going on in string theory (but, I think it would be worth a try).

In the 1950s, virtually every major university had an accelerator of its own, including MIT, Harvard, Yale, Columbia, Cornell, Princeton, Chicago, the University of Illinois, Michigan, Minnesota, Berkeley, Cal Tech, etc. The really big facilities were the $6-\mathrm{GeV}$ Bevatron at Berkeley, and the 3-GeV Cosmotron at the Brookhaven National Laboratory. I learned about accelerators at a time when the experimenters and the accelerator builders were the same people. When I was a graduate student, four of us maintained, modified and ran the MIT synchrotron. Accelerators have become much more sophisticated since then and our field has become more specialized. The accelerator builders have split off from the experimenters just as the experimenters split off from the theorists a hundred years ago.

Particle physics as practiced has changed enormously. Small experiments of two or three people that were the norm when I started out have given way to huge collaborations culminating in the ATLAS and CMS collaborations at the LHC, which have 1500 to 2000 collaborators each. These collaborations are larger than many of our laboratories.

Accelerators are huge and are built by specialists. There are very few of them. The theory has become so mathematically sophisticated that it is very difficult to keep up. An experimental physicist at the height of his or her powers (that means as a graduate student or early post doc) would have a difficult time with topology, 
knots, branes, and all of today's machinery. However, the questions really remain the same as they were when I was a graduate student: what are the fundamental entities, what determines their properties, what governs their interactions, how did it begin and how is it going to end.

Our science, like all sciences, repeatedly goes through a three-part cycle. In one phase, experiment leads theory. The discoveries come thick and fast, and no model exists to absorb and interpret them. That was the situation in the 1950s when meson and baryon resonances were proliferating and it became clear that these could not be the fundamental entities from which all matter was built because there were too many of them.

The second and perhaps most enjoyable phase is the time when experiment and theory advance rapidly together. The 1960s and 1970s were like that, when the experimenters and theorists played a kind of ping-pong game when an experiment would lead to a new theoretical interpretation which led to a new experimental test which led to a new modification of the theory. In quick order over a period of ten years, the standard model rose from deep inelastic scattering, scaling, quarks, neutral currents, $\psi / J$, the third generation, the GIM mechanism, renormalizable gauge theories, etc.

Now we are in a stressful and frustrating phase. We have an obviously incomplete model and no experiment definitively points the way toward the next step. The standard model stands uncontradicted, yet we know perfectly well that it is wrong: it has too many arbitrary constants, 18 of them if you do not include the seven more that may come from neutrino masses and a neutrino-mixing matrix. It is interesting to note that there are more arbitrary constants in the standard model now than there were resonances in the particle data book when it became the accepted view that there were too many resonances for them to be fundamental. There are other problems as well: there is not enough $\mathrm{CP}$ violation in the standard model to create the baryon asymmetry of the universe and there are potential problems with longitudinal $W$ scattering.

We are at a point in high-energy physics where we have a wonderful model with its quarks, leptons, force particles, and its $S U(3) \times S U(2) \times U(1)$ structure. All that is currently accessible to experiment has been correctly predicted by the theory (except for the 18 to 25 constants, of course). Yet there are things that the standard model cannot deal with and, at all our conferences in recent years, we have hoped to hear the experiments and theoretical ideas that will lead us beyond it.

\section{CP Violation}

Many of the advances in high-energy physics, indeed in all of science, come from the overthrow of unexamined assumptions. Such is the history of CP violation. The first assumption to go was that of parity conservation, a theoretical simplification which 
had been experimentally shown to be correct for the electromagnetic and nuclear forces. It was then assumed that it was correct for the weak interaction as well.

In the early $1950 \mathrm{~s}$, high-energy physics was struggling with the $\theta-\tau$ paradox. Experiments had turned up what seemed to be two spin-zero particles, one of which decayed into two $\pi$-mesons while the other decayed into three $\pi$-mesons. Both appeared to have the same mass to a precision of a few tenths of a percent. Lee and Yang, in their famous paper [1], analyzed what we really knew about parity conservation in the weak interactions and concluded that there were no constraints. They gave several examples of experimental tests that could settle the question, and C. S. $\mathrm{Wu}$ did one of those, studying nuclear beta decay and finding that parity was not conserved. Very soon thereafter experiments at the Columbia and Chicago cyclotrons found that parity was also not conserved in the $\pi$ - $\mu$-electron decay chain. Fitch and Cronin in their experiment of 1966 found that CP was not conserved in kaon decay, and ever since we have been trying to understand what is going on.

So far, all we know about CP violation comes from the study of the $K$-meson system. That should change next year when the asymmetric $B$-Factories at SLAC and KEK produce enough data for significant measurement of $\mathrm{CP}$ violation in the $B$-meson system. Both machines and detectors have started up very well and, at the time of this writing (November 1999), PEP-II has reached a luminosity of $1.4 \times$ $10^{33} \mathrm{~cm}^{-2} s^{-1}$, about $40 \%$ of design. KEK-B is not far behind. It is reasonable to expect that roughly $10 \mathrm{fb}^{-1}$ of data will be accumulated by the time of the year 2000 Rochester Conference in Kyoto, which should be enough to achieve an error of about \pm 0.15 in $\sin 2 \beta$ from the $\psi-K_{s}$ channel.

There are new results from studies of the $K$-meson system that address the existence of CP violation outside the CKM matrix. Both CERN (NA-48) and FNAL $(\mathrm{K}-\mathrm{TeV})$ groups have new results measuring $\epsilon^{\prime} / \epsilon$. These results are given together with their previous results in Table I. The weighted average of the results has a $\chi^{2}$ per degree of freedom of 2.8, which gives a confidence level of less than $3 \%$, an improbable result. The particle data group in such cases scales the errors to get the appropriate $\chi^{2}$ per degree of freedom, a procedure that some may object to. I do it anyway and, with the error scaled, $\epsilon^{\prime} / \epsilon$ is still not consistent with superweak interactions.

The low confidence level of these results illustrates a problem that we all should think carefully about; are we handling errors properly? The two FNAL experiments, for example, have a probability of only one in 300 of coming from a random set with gaussianly distributed errors. The Fermilab group is led by the most careful physicist I know, Bruce Winstein, and the low probability may simply be bad luck. There is, however, another possibility and that is that we don't really fully understand the error distribution functions.

All of us in high-energy physics are guilty of treating a collection of systematic errors as if they were random gaussianly distributed errors, a procedure that we know is wrong. However, we don’t know how to do it any better. We also know that 


\begin{tabular}{|l|c|c|}
\hline Experiment & $\epsilon^{\prime} / \epsilon\left(10^{-4}\right)$ & Comment \\
\hline \hline E-731 & $7.4 \pm 5.9$ & FNAL 1993 \\
\hline NA-31 & $23.0 \pm 6.5$ & CERN 1993 \\
\hline K-TeV & $28.0 \pm 4.1$ & FNAL 1999 \\
\hline NA-48 & $18.5 \pm 7.3$ & CERN 1999 \\
\hline Average & $21.2 \pm 2.8$ & $\chi^{d} /$ dof $=2.8$ \\
\hline Average & $21.2 \pm 4.7$ & Scaled Errors \\
\hline
\end{tabular}

Table 1: High-precision $\epsilon^{\prime} / \epsilon$ Measurements

even if an individual error is random and gaussianly distributed, ratios of sums and differences of such quantities may not be gaussianly distributed. There are also nongaussian tails on acceptance functions, tracking functions, etc. We need to understand this problem better but, until we do, complex experiments with many variables, complicated triggers, and many cuts in the analysis process, should perhaps be treated with some caution. Treating errors in these complicated experiments as if they were gaussian may lead us to ascribe a much higher confidence level to a conclusion than is really deserved.

\section{Neutrinos}

Certainly one of the most exciting areas of research at present is neutrino physics. It is fair to say that the results of the last decade on the neutrinos from the sun, from the atmospheric interaction of cosmic rays, and from accelerators, are changing our thinking and challenging the standard model. There are new data from Super Kamiokande (Super-K) on solar neutrinos; new data from Super-K and others on atmospheric neutrinos; and still a problem with the Los Alamos experiment (LSND) which doesn't seem to fit well with our current prejudices.

Before discussing the present situation, I want to mention two people who are not currently involved in the program, but who played an absolutely critical role in the evolution of neutrino physics. The first of these is Dr. Raymond Davis, Jr., now retired, of Brookhaven National Laboratory. Ray Davis had an idea that at first seemed impossible, to detect neutrinos from the sun as a way to find out about the nuclear physics of the solar cycle. As far as I can tell, the original idea of using the chlorine-argon inverse beta-decay reaction to detect neutrinos goes back to a paper by Bruno Pontecorvo [2] written in 1946. In that paper, Pontecorvo discussed ways to detect neutrinos, including neutrinos from the sun. He dismissed the solar idea because the flux would be too small for a one-cubic-meter detector which was the 
largest that he could think of.

Ray Davis thought much bigger. In 1955, he began working at Brookhaven with a 1000-gallon chlorine detector. He found no events because of the small size of the detector but, with this apparatus, he perfected the argon-chlorine radio-chemical separation techniques that allowed his later experiments to succeed. In the early 1960s, he began construction of a 100,000-gallon detector at the Homestake Mine in South Dakota and began collecting data in 1967. From the very beginning his results indicated that the flux of neutrinos from the sun was less than that predicted by the solar models. This was greeted at first with considerable skepticism, but over the years Davis' results began to be taken more seriously and, with the results of the SAGE and GALLEX experiments, the reality of the neutrino deficit has been fully accepted. Davis' work opened this field.

The second person I want to mention is Professor Masatoshi Koshiba, now retired from the University of Tokyo. In 1979, Koshiba proposed the construction of the huge water Cerenkov counter that became known as the Kamiokande Detector. Construction was completed in 1983 and initial experiments focused on the search for proton decay. By 1985 the simplest SU-5 version of grand unified theories had been ruled out. With the addition of an outer veto layer to the detector beginning in 1984, Kamiokande became capable of detecting neutrinos as well. Kamiokande measured the solar neutrino flux, confirming Davis' result, and also detected neutrinos from supernova SN1997A, opening up a new field of neutrino astrophysics. Even before much in the way of results from Kamiokande had come in, Koshiba proposed, in 1983, the construction of the very much larger Super-K. Under his leadership the project was approved just before his retirement from the University of Tokyo. The results from Super-K are what are generating all the excitement about atmospheric neutrinos.

\begin{tabular}{|l|c|c|}
\hline Experiment & Threshold Energy & Ratio to Solar Model \\
\hline \hline Homestake Mind & $817 \mathrm{keV}$ & $0.33 \pm 0.03$ \\
\hline SAGE & $235 \mathrm{keV}$ & $0.52 \pm 0.07$ \\
\hline GALLEX & $235 \mathrm{keV}$ & $0.59 \pm 0.06$ \\
\hline Kamiokande & $7 \mathrm{MeV}$ & $0.54 \pm 0.07$ \\
\hline Super-Kamiokande & $5.5 \mathrm{MeV}$ & $0.48 \pm 0.02$ \\
\hline
\end{tabular}

Table 2: Results of Solar Neutrino Experiments

The results of the solar neutrino experiments are summarized in Table 2. The SAGE and GALLEX experiments with their 235-kV neutrino energy threshold are the only ones that are sensitive to the proton-proton part of the solar cycle where almost all of the energy of the sun is produced. The Homestake experiment (Ray Davis') with its $800-\mathrm{kV}$ threshold is sensitive to the beryllium-7 line plus the boron- 8 
continuum. The Kamioka and Super-K experiments are sensitive only to the boron-8 continuum. The surprising, to me, result is the Homestake experiment which gives a ratio to the solar model of one-third, while the other four give a ratio of one-half. Any explanation of these results is complicated by the required energy dependence of the neutrino depletion process needed to account for all of the results. Super-K also reports a statistically marginal distortion of the neutrino spectrum compared to the standard solar model, and a 1-1/2 to 2 standard deviation day-night effect. Neither of these two effects is as yet of the significance required to be taken seriously.

Figure 1 is from Y. Suzuki's talk [3] at this conference summarizing the solar neutrino situation within the framework of neutrino oscillations. The upper part of the figure gives the allowed regions in the MSW model (a resonant conversion of the electron-neutrinos into another species of neutrinos) while the lower figure gives the allowed regions for pure vacuum oscillations. It is worth noting that if there is a systematic effect in the Homestake experiment, and all of the results were to be consistent with an energy-independent reduction of the solar neutrino flux, the large mixing-angle solution would be allowed with any $\Delta m^{2}$ from about $10^{-9}$ to $10^{-4} \mathrm{eV}^{2}$.

The LSND experiment has been a problem since the first results were presented in 1995. It is the only experiment that purports to show the conversion of muon neutrinos into electron neutrinos with a large $\Delta m^{2}$. The first results appeared to be in conflict with other experiments, particularly the KARMEN experiment at the Rutherford Laboratory. There is more data now, and as DiLella [4] showed in his talk, there appears now to be a narrow range where the results of all of these experiments are consistent. Figure 2 shows the present situation.

The LSND experiment uses muon antineutrinos arising from the decay of mesons produced at the LAMPF $1-\mathrm{GeV}$ proton accelerator. If these muon antineutrinos oscillate into electron antineutrinos, these can interact with protons in the detector tank (behind a thick shield) to produce positrons (20- to $60-\mathrm{MeV}$ cuts) plus a neutron which is detected after a delay by a neutron-captured gamma ray. Electron neutrinos, which can be produced directly in the beam, can also interact in the detector, but they interact with carbon and there is no delayed neutron-captured gamma ray. The experiment analyzing all the data from 1993 through 1998, claims to see an excess of positrons in coincidence with a delayed neutron capture of $40 \pm 9$ events. That is certainly statistically significant, even with the statistical skepticism I evidenced earlier, but there are still many doubters and I include myself among the doubters.

Figure 3 is from the LSND paper [5] and shows the distribution of accidental events in their detector tank. There is clearly a large excess of such events at the bottom front of the tank and this can only come from neutron leakage under their shield. This is a "beam on" background that the experimenters eliminate by focusing on a fiducial region toward the back and above the bottom of the detector.

Neutron diffusion is much less of a potential problem in the KARMAN experiment, because their machine produces a $10 \mu s$ beam pulse while LAMPF produces a 600 


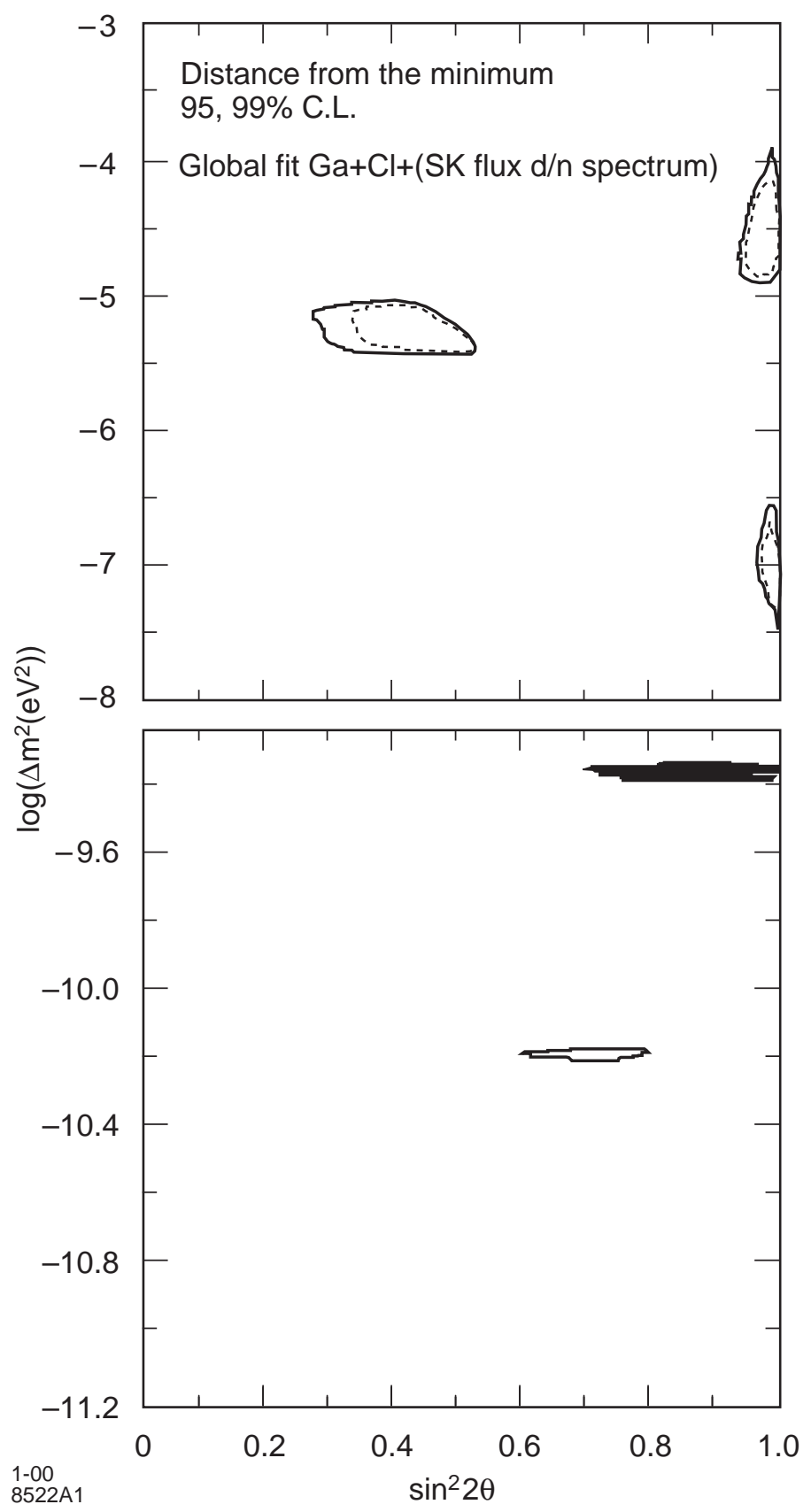

Figure 1: Global fit to the solar neutrino data. [3] 


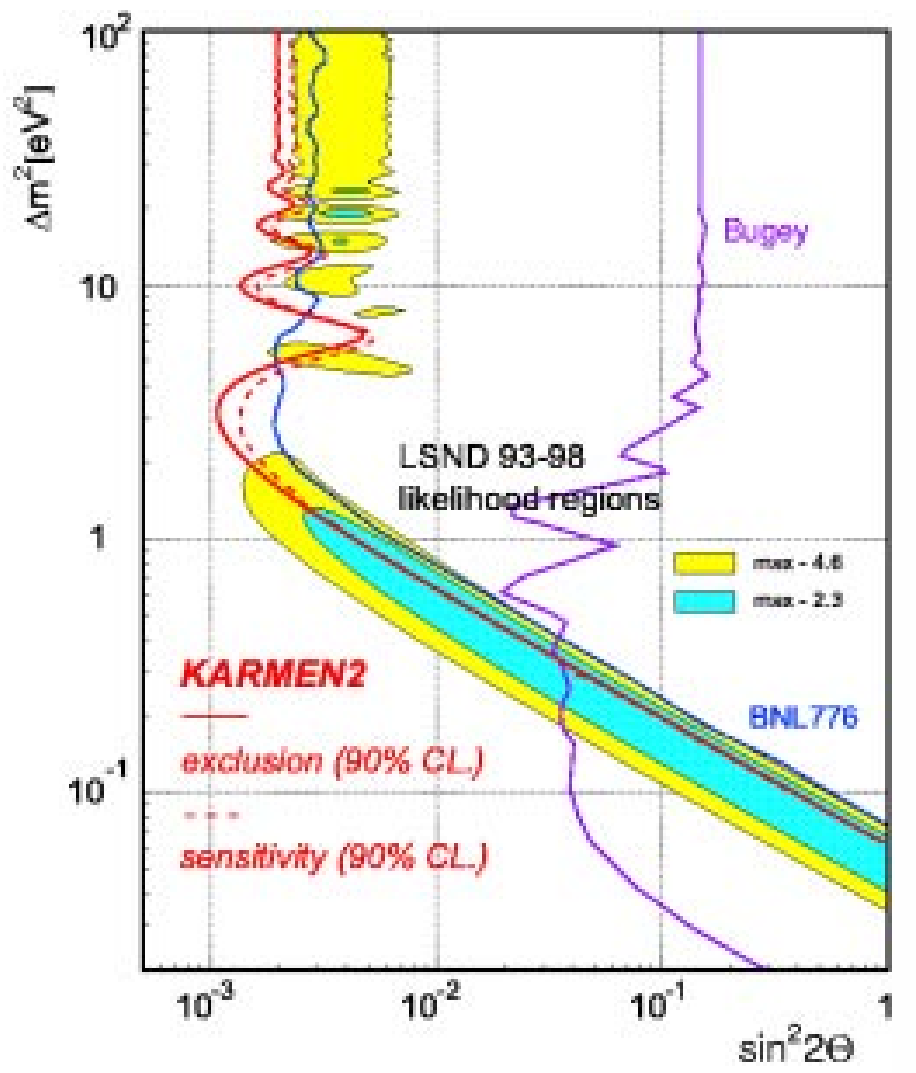

Figure 2: Electron neutrino experimental results. LSND is not in conflict with other experiments in a band from $\Delta m^{2}$ of $0.3 \mathrm{eV}^{2}$ and $\sin ^{2} 2 \theta$ of $3 \times 10^{-2}$ to $\Delta m^{2}$ of $1 \mathrm{eV}^{2}$ and $\sin ^{2} 2 \theta$ of $1.5 \times 10^{-3}$. [4]

$\mu s$ pulse. Neutron diffusion under the shield is a slow process and so KARMAN can gate most of this out with a relatively narrow time window around the beam pulse.

This experiment will have to be done again and it will be done again at FNAL in the MiniBooNE. The MiniBooNE detection limits are shown in Fig. 4 and the first results should be available in the year 2003. We have to wait.

The most exciting news on the atmospheric neutrino front comes from the SuperK data. Figure $5[6]$ shows an apparent disappearance of muon neutrinos as a function of zenith angle. Muon-like events are strongly depleted when they are generated by neutrinos passing through the entire earth compared to those generated by neutrinos coming down through the relatively thin covering of the Super-K detector. The allowed mass difference region assuming $\nu_{\mu}$ to $\nu_{\tau}$ oscillations is shown in Fig. 6. The Macro and Soudan experiments see a similar effect though with much looser mass constraints. Note that the Super-K/Macro/Soudan data alone do not tell us what 

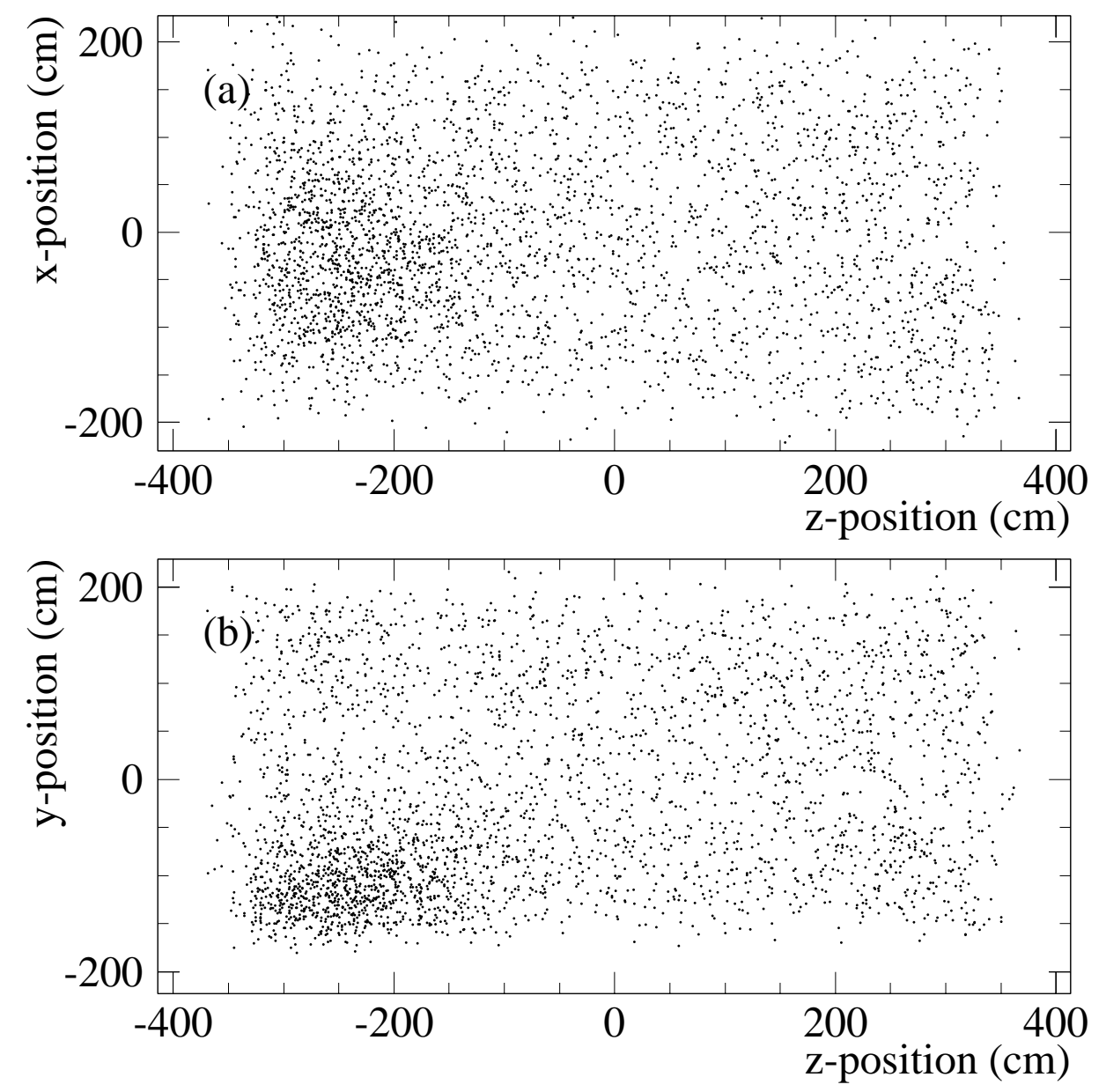

Figure 3: Spatial distribution of accidental background events in the LSND detector.

happens to the muon neutrinos, they only tell us that they disappear. Muon neutrinos could go to sterile neutrinos that don't interact; $\tau$ neutrinos that can only generate neutral current events since all the neutrinos are below $\tau$ production threshold; or even to some extent into electron neutrinos since it is hard to tell an enhancement of electron neutrinos from a shortage of muon neutrinos in this data.

The CHOOZ reactor experiment, in conjunction with the Super-K data, imposes the tightest restrictions on muon neutrino electron neutrino mixing. Fogli et al.[7] analyzes Super-K and CHOOZ data together (Fig. 7). The shaded regions give the $90 \%$ and $99 \%$ allowed regions for the two experiments. The analysis done is done in the "dominant mass" mode and Super-K alone allows a quite large mixing of electron neutrinos. Super-K combined with CHOOZ, however, says that if there are 


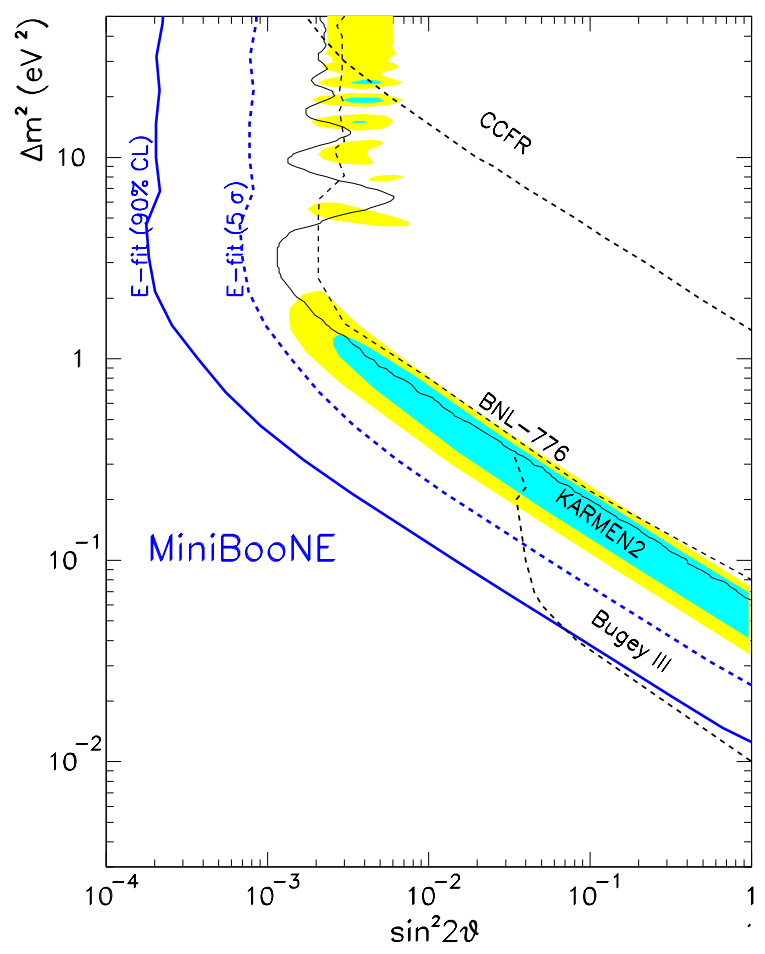

Figure 4: Expected range of the MiniBooNE experiment in the electron neutrino $\Delta m^{2}$ versus $\sin ^{2} 2 \theta$ plain superimposed on the present results. Data taking is scheduled to begin in 2002. [4]

oscillations, the muon neutrino oscillates almost entirely into tau neutrinos. Another reactor experiment, the Palo Verde experiment, has only been running for a relatively short time but already confirms the CHOOZ result in the mass range favored by Super-K.

Before discussing the next generation of experiments, it is useful to pause to summarize what we know:

1. Neutrinos from the sun are below the predictions of the standard solar model. All of the experiments integrate over neutrino energies above some threshold. The SAGE and GALLEX experiments, with a threshold of $235 \mathrm{keV}$, are a factor of two below the expectations; the Homestake experiment, with a threshold of $817 \mathrm{keV}$, is a factor of three below expectation; and the Kamiokande and Super-K experiments, with thresholds of 5 to $7 \mathrm{MeV}$, are a factor of two below expectations. 


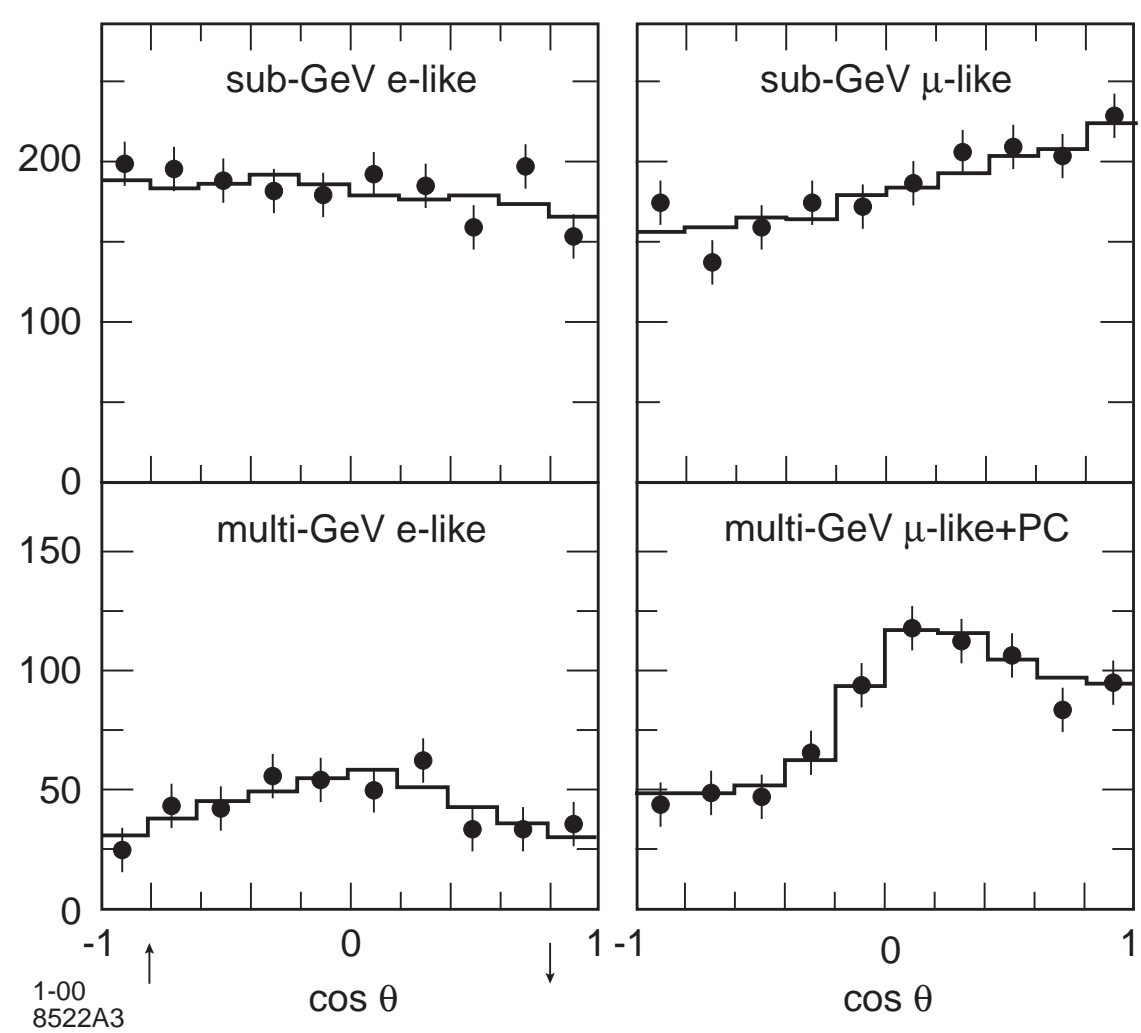

Figure 5: Zenith angle distribution of the Super Kamiokande data for electron-like and muon-like neutrino events. The muon-neutrino-like events are strongly depleted for upward-going neutrinos. [6]

2. The atmospheric neutrino experiments clearly see an azimuthal dependence of the muon-neutrino/electron-neutrino ratio indicating a decrease of muon neutrinos coming 12,000 km through the earth.

3. The LSND experiment claims to see muon-neutrino to electron-neutrino conversion, but this experiment needs confirmation.

4. The CHOOZ experiment is the most sensitive of the reactor experiments and sees no loss of reactor-generated electron neutrinos over distances in the order of kilometers.

Taking all of this together, the favored explanation is neutrino-flavor oscillations plus the MSW effect. However, this is not the only explanation consistent with the data we have so far. For example, Barger et al.[8] proposed to explain the data with a mixture of neutrino decay and the MSW effect. Gonzales-Garcia et al. [9] hypothesized 


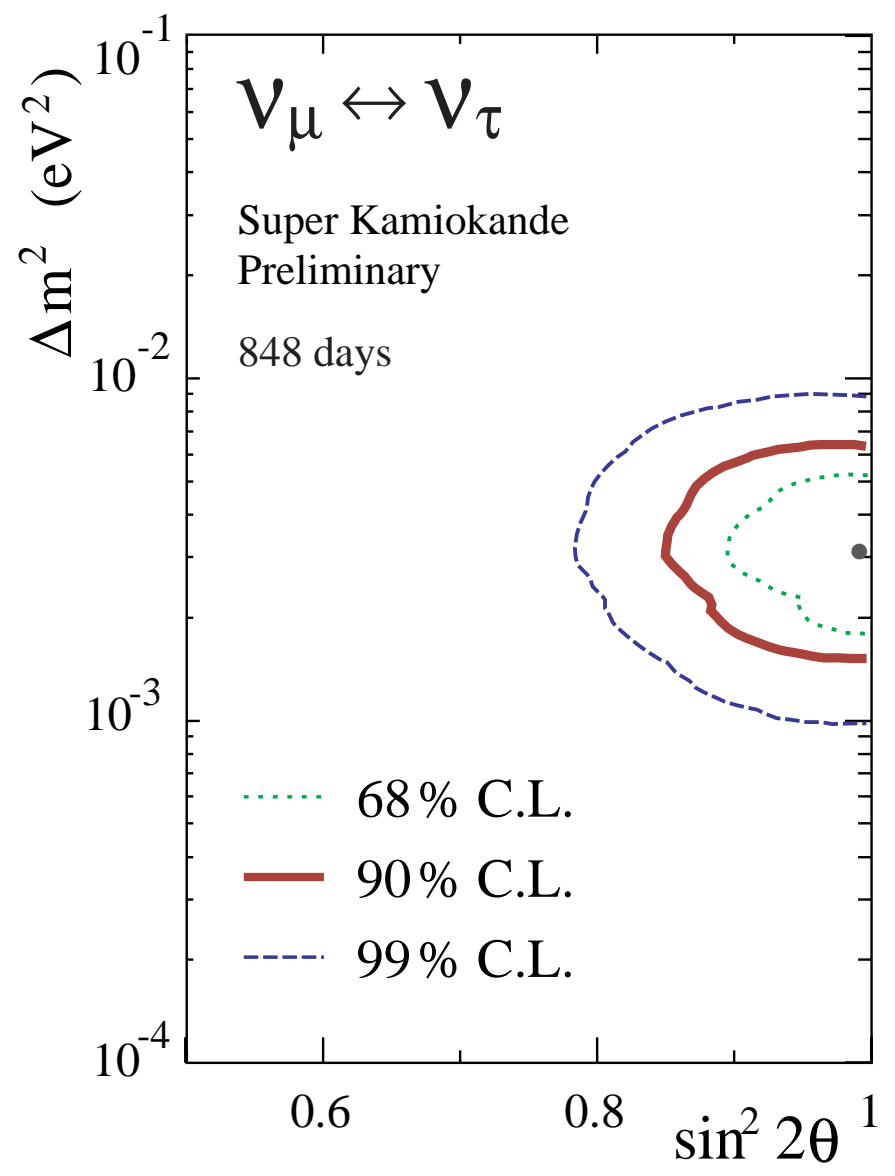

Figure 6: Allowed $\Delta m^{2}$ and $\sin ^{2} 2 \theta$ from the Super Kamiokande data assuming $\mu-\tau$ neutrino oscillations.[6]

flavor changing in neutral current interactions of neutrinos. Both of these alternative hypotheses invoke new physics but so does the favored explanation. The job of the next generation of experiments is to sort all of this out.

On the solar neutrino front, we will certainly get more data from Super-K and data from two new experiments, SNO (already operating) and Borexino (due to start up in one to two years). I doubt that more statistics from Super-K will tell us anything new, but the other two experiments certainly will. Of particular interest to me is Borexino, which should be able to resolve the $\mathrm{Be}^{7}$ line and so pin down any energy dependence with some precision.

The Kamlands reactor experiment in Japan is also important. Its electron neutrinos come from twelve nuclear reactors at an average distance of $150 \mathrm{~km}$. Kamlands 

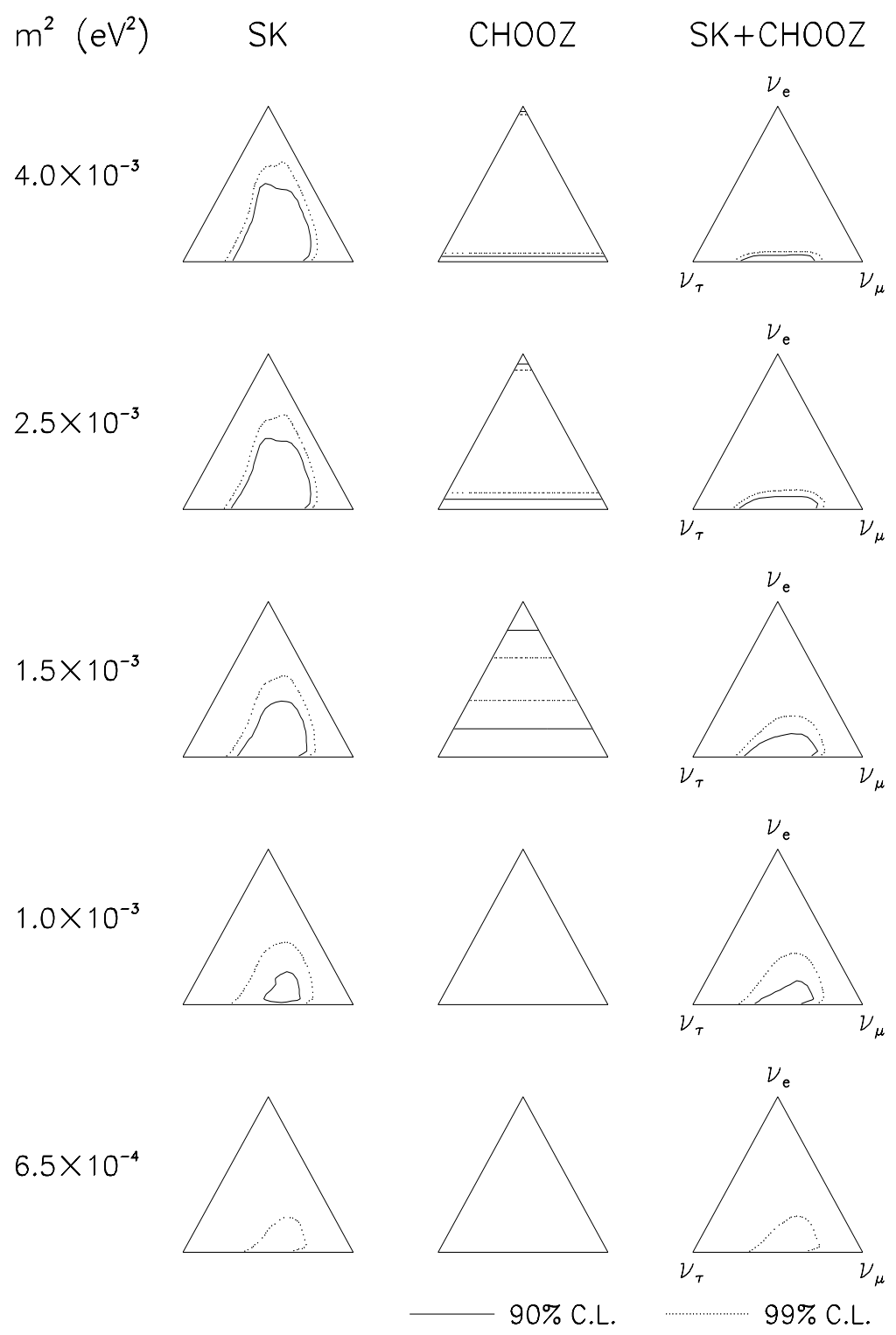

Figure 7: The Fogli, et al., analysis showing how the CHOOZ electron-neutrino disappearance limit together with the Super Kamiokande results limit the electronneutrino/muon-neutrino coupling. [7] 
is a conversion of the old Kamiokande experiment and it should have a sensitivity to electron-neutrino oscillations down to $\Delta \mathrm{m}^{2}$ less than $10^{-5} \mathrm{eV}$, covering the large mixing-angle MSW solution to the solar neutrino deficit.

Super-K will get much more data on atmospheric neutrinos. Of particular interest would be data around 90 degrees from the vertical. If oscillation is the answer and the mass difference is around Super-K's central value, their sub-GeV and multi-GeV samples will show different behavior in this region. The $0.5-1 \mathrm{GeV}$ neutrinos would be near their first oscillation minimum at 200-km distance, while the above $1-\mathrm{GeV}$ neutrinos would show little reduction. Super-K may not have enough flux, consistent with the required angular resolution, to do this analysis.

The K2K experiment takes muon-neutrinos from KEK's 12-GeV proton synchrotron to the Super Kamiokande detector and has just begun taking data. This is a disappearance experiment using muon neutrinos of less than $2 \mathrm{GeV}$. If the mass difference is above about $2 \times 10^{-3} \mathrm{eV}^{2}$ they should clearly see an effect in two to three years of data taking. This would be the first independent check of the Super-K result. In principle it is possible for the $\mathrm{K} 2 \mathrm{~K}$ experiment to definitively confirm the oscillation hypothesis. At the central value of the Super-K mass difference, $500 \mathrm{MeV}$ neutrinos oscillate away and $250-\mathrm{MeV}$ neutrinos oscillate back. However, the neutrino flux and the Super-K energy resolution are probably not good enough to see this, but it would be wonderful if they were.

I have already mentioned the MiniBooNE experiment. It supplies a definitive check on LSND and we will have to wait a few years for results.

The long baseline experiment, MINOS, is under construction at Fermilab and at the Sudan Mine. It is expected to begin data taking in the year 2003. MINOS is capable of seeing tau neutrino appearances over most of the mass difference range allowed by Super-K. Under MINOS' conditions, neutrinos of $2 \mathrm{GeV}$ will oscillate away and $1 \mathrm{GeV}$ will return. If I were running the experiment, I would certainly tune the beam to low-energy neutrinos for the first few years.

CERN and the Gran Sasso Laboratory in Italy are discussing a European longbaseline experiment. The beam and the distance are very similar to the MINOS experiment. If this experiment is approved, I hope the apparatus is sufficiently different from MINOS to make the investment worthwhile.

Finally, there is much discussion of the potential of a muon storage ring as a high-intensity neutrino source. Such a facility would be much simpler to build than the muon collider that has been discussed for the past few years, but there are issues that couple the storage-ring design to the experiment. These issues have not yet been fully explored. Figure 8 [10] shows what the excitement is all about; lots of neutrinos and greatly improved sensitivity. Figure 9 shows the problems; the storage ring is a mixed source of, for example, muon-neutrinos and electron-antineutrons.

The muon and electron neutrino spectra from unpolarized muons in the storage ring (much easier for the machine builder) are not that different, and only a detector 


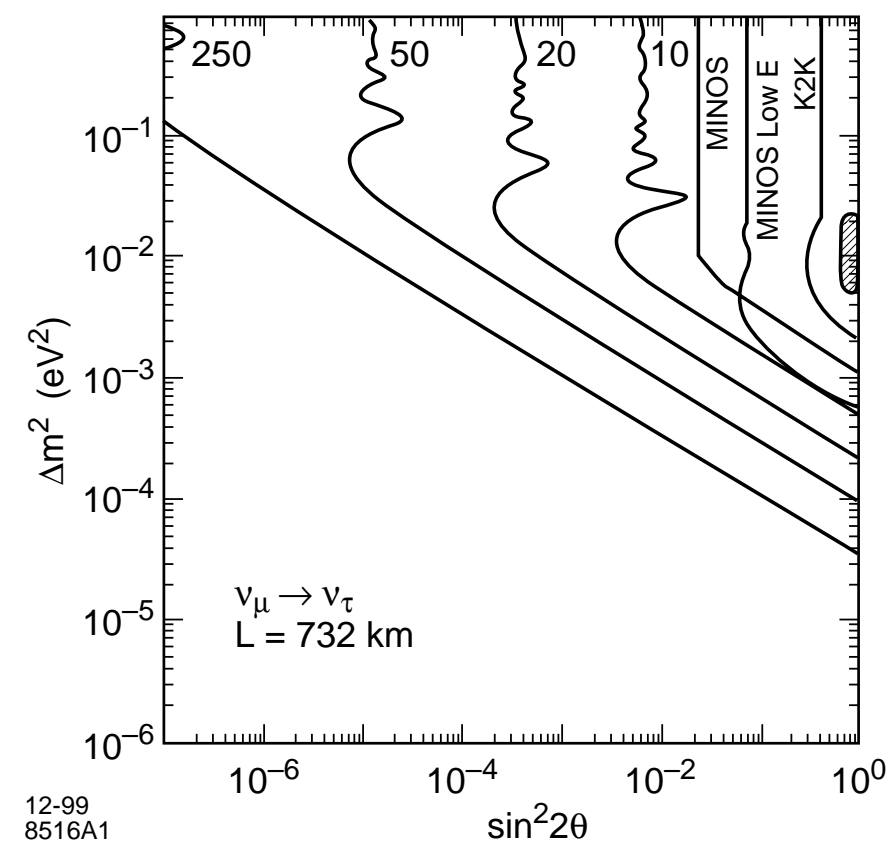

Figure 8: The Barger, et al.[8], calculation of the limits that could be placed on $\nu_{\tau}$ appearance using a muon storage-ring neutrino source of various muon energies.

with really good energy resolution could separate them on a statistical basis. To complicate the situation further, if the muon beam in the storage ring has a transverse momentum comparable to the muon mass (this gives the highest flux), then the two spectra are smeared further making the two types still harder to separate.

Polarization of muons in the storage ring allows the electron neutrinos to be tuned all the way to zero (in principle). However, the best polarization that the machine designers have come up with so far is in the range of $20-30 \%$ and even achieving this costs considerably in complexity and somewhat in flux in the machine.

Groups in the U.S. and in Europe are working on the machine and on the physics. They need to work more closely together to better understand the trade-offs between the experiment and the machine so that both can be properly optimized in order to carry out the required physics program.

\section{Accelerators}

At this conference, J. Lykken [12] has spoken of the physics potential of the next generation of proton and electron accelerators, and G. A. Voss [13] has spoken of the state of technologies. I can, therefore, be brief. The next big machine, the LHC, 


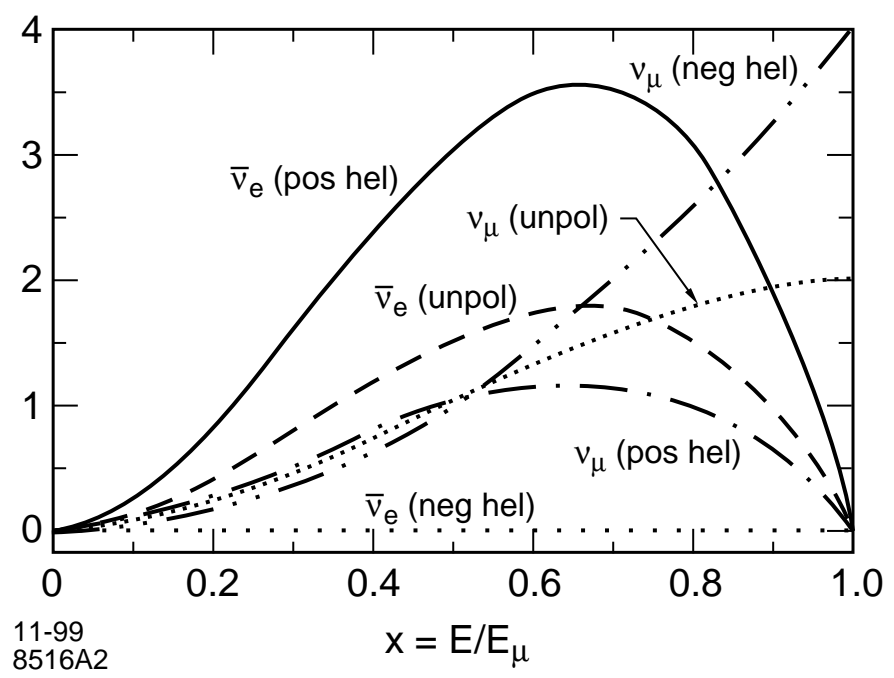

Figure 9: Normalized neutrino flux versus fractional neutrino energy at zero degrees for various negative muon helicity states. [11]

is under construction at CERN and is close to being the first world project. Contributions to the accelerator and detector have been made by many nations outside the group of CERN member states. The LHC will have an energy of $14 \mathrm{TeV}$ in the proton-proton center of mass, a luminosity of $10^{34} \mathrm{~cm}^{-2} s^{-1}$ and a mass reach of about $1 \mathrm{TeV}$. Operations are expected to begin in the year 2005.

The two main experiments ATLAS and CMS will each have 1500 to 2000 collaborators. The size of these collaborations is unprecedented and presents difficult organizational problems in getting ready and new sociological problems in operation. In the 500-strong collaborations of today, we already have a bureaucratic overlay to the science with committees that decide on the trigger, data analysis procedures, error analysis, speakers, paper publications, etc. The participating scientists are imprisoned by golden bars of consensus. While we have survived so far and preserved some opportunity for scientific initiative, this will become more difficult as the collaborations grow to three times the size of today's largest. This needs thinking about and talking about, but is a topic for another time.

The theorists tell us what they want us to find with the new accelerators, the source of electroweak symmetry breaking. But, that is not the experimenters' job. Our job is to find out what is really there and, in this case, that means to find if there is any electroweak symmetry making. The experiments are difficult and the detectors are complex, expensive devices. Data rates, particularly at proton colliders, are enormous and there is no way to digest it all. Complex, multi-tiered trigger 
systems are needed to reduce the flood of data coming from the machine by a factor of 10 million or more so that our computer systems can handle the load. Those events that do not pass the trigger screen are discarded.

There is a danger here. Will we set up the experiments that can only find what we expect to find? Some years ago I asked the spokesperson for a large experiment if they could see a magnetic monopole that decays into a large number of 0.5 to 1 $\mathrm{GeV}$ gamma rays (a model that was plausible at the time). The answer was "no"; the trigger screened out such isotropic events that had no jet-like clustering and no transverse energy unbalance. While this particular problem is fixed, there is a lesson here. Be prepared and allow some of the trigger to be dedicated to what may currently be unfashionable.

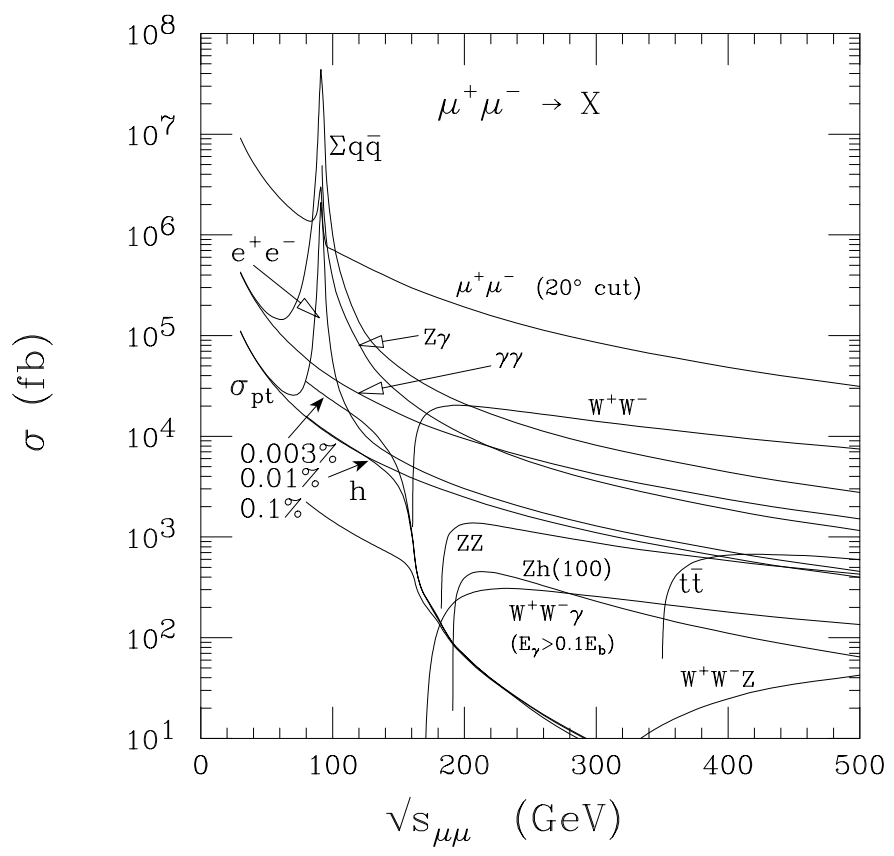

Figure 10: Lepton/anti-lepton cross-sections versus center-of-mass energy. All crosssections except for $s$-channel Higgs production are the same for $e^{+}-e^{-}$and $\mu^{+}-\mu^{-}$ colliders.

With the LHC well underway, the next accelerator facility will be an electronpositron linear collider. Lykken, in his presentation, told of the physics potential and how the linear collider seems a necessary compliment to the LHC. Electron-positron machines have one advantage over proton colliders: all of the cross-sections are within a few orders of magnitude of each other (Fig. 10) and it is still possible to record all of the events that occur. Beams in linear colliders can be polarized and this is a 
great help in untangling the physics. Research and development is nearly done and it is hoped that construction of a new facility can start in 2004 or 2005 and the physics program can begin in 2010. The machine will be costly and will surely have to be built by worldwide collaboration.

A critical issue not yet settled is the initial energy for a new facility and what, if any, expansion capabilities should be built into the initial design (spending some extra funds now can save time and costs later for an energy increase). There seems to be a convergence on $500 \mathrm{GeV}$ as an appropriate initial center-of-mass energy with the potential to expand to something in the 1 to $1.5 \mathrm{TeV}$ region. There is a worldwide physics study currently underway with coordinators in Asia, Europe and North America. Whatever the initial energy, this project will be expensive and will only happen if the entire high-energy physics community gets behind it.

There has been much talk about the possibility of muon colliders. The attraction of the muon collider is also its biggest problem. Synchrotron radiation, which may limit the performance of multi-TeV electron-positron linear colliders, is absent in a muon collider. This absence requires the development of a new method of emittance damping to shrink the phase space in which the muons are produced so that high luminosity can be obtained in the collision. This will require a major R\&D effort and one is being planned. It will not be simple.

The latest story on the state of the R\&D in systems design is given in Ankenbrandt et al.[14] The luminosity of such a collider appears not to be interesting until a multi$\mathrm{TeV}$ energy is reached. The indicated low-energy option has luminosity less than that of LEP-II, while the medium-energy example has luminosity less than the linear collider. All of the cross sections for muon colliders are the same as those for an electron-positron collider except for an s-channel process coupled to mass, Higgsboson production. Here also the muon collider is not as effective as the electron collider. The rate for the production of $100-\mathrm{GeV}$ mass Higgs in the muon collider is given in the reference above to be about 4000 per year. The $500-\mathrm{GeV}$ electronpositron machine produces five times that rate through the Higgs plus $Z^{0}$ channel and the $W$-fusion channel.

The muon source itself is expensive. Current studies use a 4-MW proton source to produce the muons, four times the power of the SNS spallation neutron source which is estimated to cost $\$ 1.3$ billion. Perhaps this can be done for less by the upgrade of one of the existing proton machines, but any real test of the muon collider concept will require a physics program that justifies the large cost. Perhaps the muon storage rings being discussed as neutrino sources can supply the justification for a real trial of the technology. 


\section{Non-Accelerator Experiments}

Non-accelerator experiments are playing an ever more important role in high-energy physics; witness the time spent at this conference on Super Kamiokande neutrinos, cosmic microwave background fluctuations, and supernova distributions. These experiments allow tests of our theories not possible with accelerators. More such experiments are coming, such as:

- The Sloan Digital Sky Survey which will map the large-scale structure of luminous matter in the universe to much larger $z$ than now. This information is critical to test cold versus warm versus hot dark-matter scenarios.

- The AUGER ultra-high energy cosmic ray experiment which will get considerably more information on cosmic rays of mysterious origin with energies beyond the cutoff from interaction with the $3^{\circ} \mathrm{K}$ microwave background radiation.

- The Whipple, Hegra, Egret, GLAST experiments, etc., looking for an explanation of the origin of ultra-high-energy cosmic gamma rays.

- Neutrino observatories, under the ice and under the oceans.

- Follow-ons to COBE and the Supernova Cosmology Project that will give clues to the basic structure of the universe.

Some in high-energy physics are concerned that such experiments may drain funds from accelerator-based activities that have dominated high-energy physics for many decades. That may be so, but high-energy physicists should go where the high-energy physics is, whether it be in space, underground, underwater, or with accelerators. There is more to high-energy physics than the Higgs boson, and collaborations with the astrophysicists and cosmologists are more likely to broaden rather than narrow support for the things we are interested in through collaborations with new (to us) areas of science.

Two particularly important programs have been discussed at this conference, the cosmic-microwave-background radiation and the supernova search. The microwavebackground radiation experiments measure the fluctuations in temperature (on the order of $10^{-5 \circ} \mathrm{K}$ ) as a function of the angular scale of the fluctuations. The pattern of these temperature fluctuations is a critical test of inflation, which ten years ago was thought to be an untestable metaphysical concept. It is testable now (see, for example, C. Contaldi, et al.[15])

The experiment looks back to about 300,000 years after the big bang when the temperature in the early universe had dropped to the point where electrons could form hydrogen atoms (see Fig. 11). At that time the mean-free path of light changed from very short to very long as free electrons were bound to atoms; metaphorically 


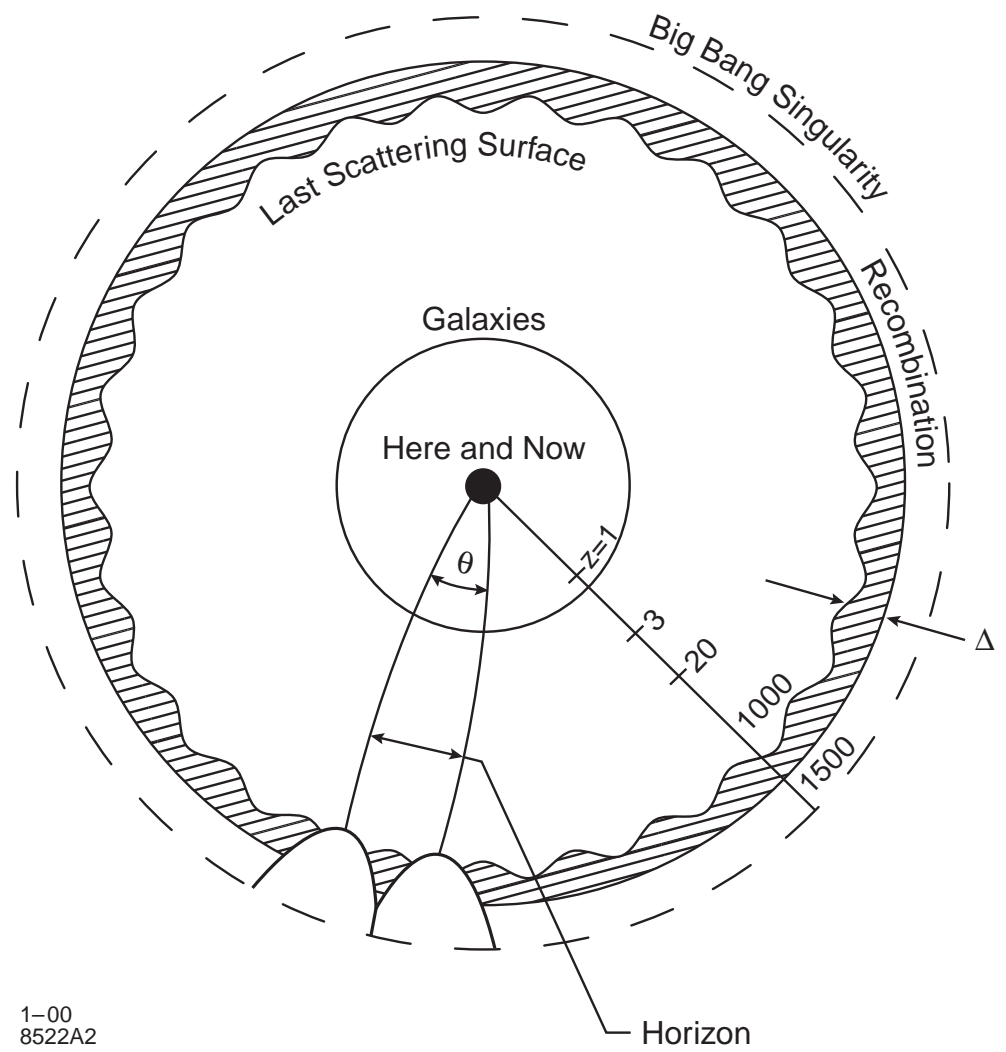

Figure 11: Schematic of the source of the fluctuations in temperature of the cosmic microwave background radiation (from the COBE website at Lawrence Berkeley National Laboratory-www.lbnl.gov).

the universe changed from foggy to clear. That light has been red- shifted by the Hubble expansion of the universe to become the $3^{\circ} \mathrm{K}$-background microwave-background radiation. Cold spots cooled early and hot spots later. The expansion leads to the fluctuations in temperature in the microwave background.

The available data are summarized in Fig. 12, which plots the power spectrum of the fluctuations versus spherical harmonic number. The information needed for test of cosmology occurs at high-harmonic numbers and the data is not precise enough yet to constrain the models. Over the next five years two new experiments will be launched, the MAP satellite by NASA, and the Planck satellite by the European Space Agency. These experiments are designed to be remarkably precise. The expected errors at large $\ell$ are expected to be comparable to the width of the lines in Fig. 12 up to $\ell \approx 1000$ for MAP and 2000 for Planck. Beyond these, the errors blow up 


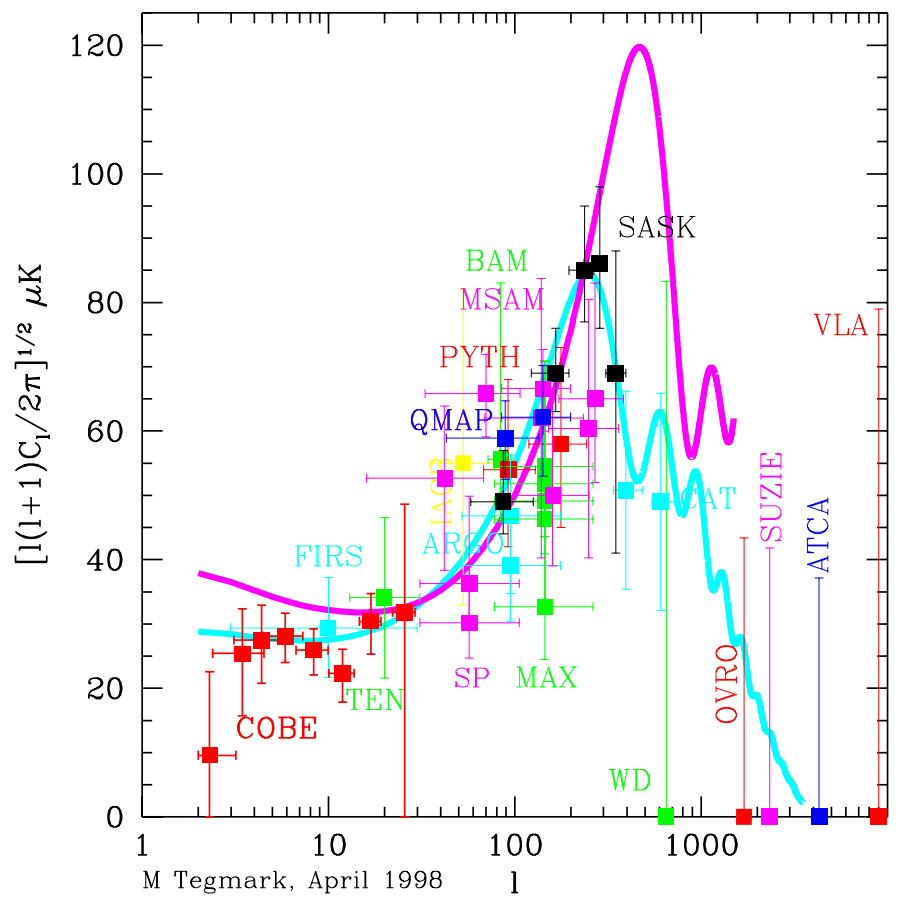

Figure 12: From Turner and Tyson. [17] Summary of current measurements of the power spectrum of CMB temperature variations across the sky against spherical harmonic number $\ell$ for several experiments. The first acoustic peak is evident. The lower curve, which is preferred by the data, is a flat Universe $\left(\Omega_{0}=1, \Omega_{M}=0.35\right)$, and the dark curve is for an open Universe $\left(\Omega_{0}=0.3\right)$ [courtesy of M. Tegmark].

very rapidly. It requires extraordinary precision to distinguish between cosmological models and, when both satellites are up, I'll believe the data from two independent satellites with two independent instruments.

Perlmutter [16] discussed the supernova cosmology project which addresses one of the most fundamental questions in physics, the constancy of the expansion rate of the universe. The results seem to say that it has not been constant, that the matter density of the universe is less than the critical density expected, and that there seems to be a cosmological constant in general relativity.

The experiment uses type I-a supernovas as standard candles, measuring the apparent brightness (distance by $R^{-2}$ ) and red shift (distance by Hubble expansion). In an unscientific sample of 15 high-energy physicists, I found only one who understood how type I-a supernovas worked and what generates the light, and so I will briefly digress to tell you about it. 
Type I-a supernovas are thought to come from white dwarf stars in binary systems that, over time, accrete matter from their companions until they reach a critical mass (1.4 solar masses). At that point, the white dwarf collapses and explodes as a supernova. Neutrinos escape immediately (SN1987A, for example). The debris cloud is heated by the decay of radioactive nickel (the minimum of the nuclear- binding energy curve), but the light is trapped because the cloud is optically opaque. As the cloud expands, its optical thickness decreases $\left(R^{-2}\right.$ for a uniform sphere, and $R^{-1}$ for a shell), and the light intensity rises. It falls with the decay of the radioactive heat source. Type I-a supernovas are rare, occurring about one per 500 years per galaxy. The collaboration has collected fifty of them out to a $z$ of 0.8 .

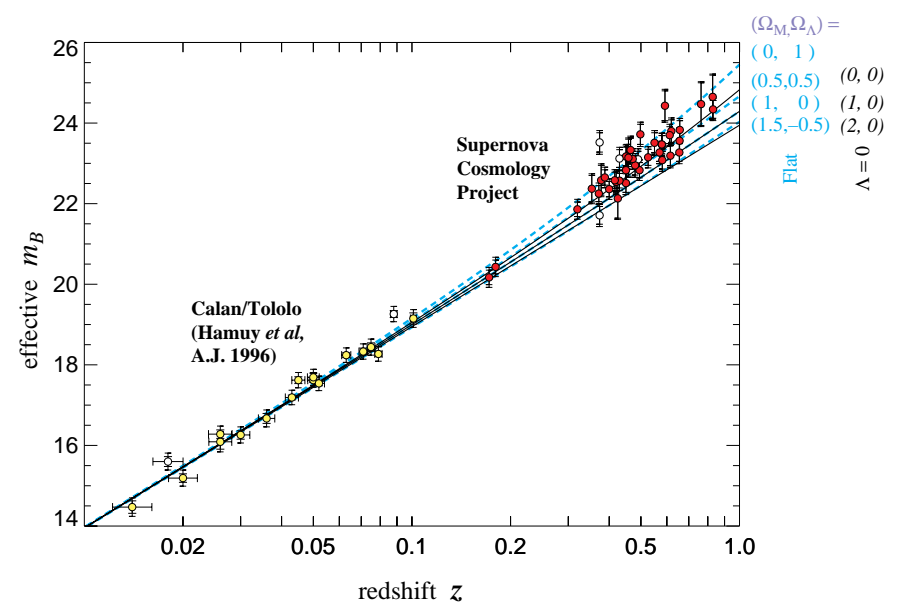

Figure 13: Effective magnitude versus redshift for Type 1-a supernova.

The data are shown in Fig. 13 that plots the apparent magnitude versus red shift. The data clearly deviate from the straight line expected for a flat universe with a matter density equal to the critical density. Perlmutter, et al.[16] find that for a flat universe the matter density is $0.28 \pm 0.085$ (statistical) \pm 0.05 (systematic) of that expected for a flat universe. The rest has to be made up by a cosmological constant.

Figure 14 shows the results in the matter-density/cosmological-constant plane. It is very far from being consistent with what we all had been assuming for many years: a matter density equal to one and a cosmological constant of zero. Personally, I would like to see this analysis done in a slightly different fashion. Since the universe obviously has some matter in it, the matter-density should be constrained to be greater than zero. This constraint will rotate the error ellipse and shift it somewhat.

The real question about the analysis has to do with the assumption that Type 1-a supernova are really standard candles, i.e., do supernovae that exploded six or seven 


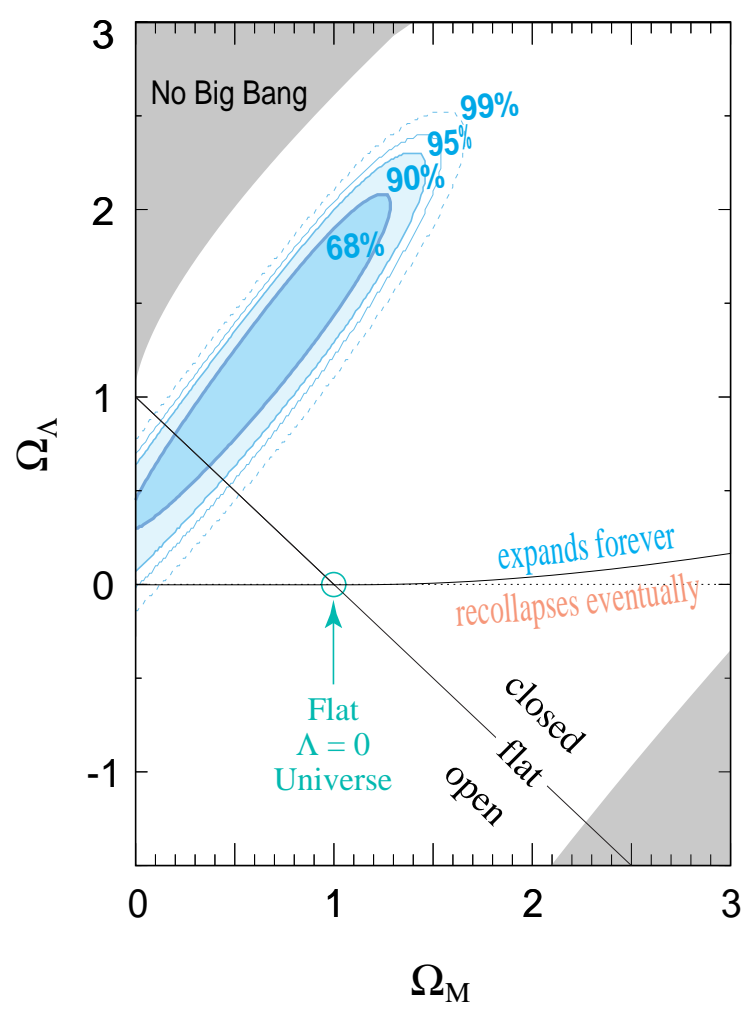

Figure 14: Cosmological versus matter densities in units of the flat universe critical density. [16]

billion years ago $(z=1)$ give the same light output as those that explode today? Stars formed recently have more heavy elements in them than did stars formed many billions of years ago, and I don't know of any studies that look at what effect if any such a systematic difference in composition might have on light output. The collaboration has clearly gone through the detailed analysis of systematic errors, but I am not sure that I have high confidence in the systematic error quoted of 0.05 on the mass density. A systematic shift of one-quarter to one-half magnitude at $z=1$ would move the cosmological constant to zero. The data are superb, but the conclusions may not be. The collaboration will be collecting much more data and have proposed a dedicated satellite to extend the data out to a $z$ of two to three. If I had the money, I would give it to them-this is really important. 


\section{Theory}

I want to talk about theory more philosophically than technically because I think it important that experimenters understand theory better so that they do not become mere technicians for those espousing the latest theoretical fad. Historically, advances in theory have synthesized data, accommodated previous theory as a special case, and simplified our view of the world. We have a bias toward elegance (in the eye of the beholder, of course) in the choice of theories and that has served us well in the past. The more mathematically and conceptually elegant theories are the ones that tended to survive.

All theories are born under-constrained in that there are always alternatives that compete in the contest with experiment, with the losers consigned to the dustbin. The sociologists of science would say that our theories are "socially constructed." That is certainly true initially but our theoretical models are continually tested and the "social constructs" that don't pass are discarded; though sometimes it takes a long time. Aristotle and Democritus had different views on whether matter was continuous or atomic in nature. Although there was no evidence to decide between these two views, Aristotle won, and for 2000 years it was believed that matter could be continuously subdivided and that there were no such things as atoms. We all believe that we are much more critical these days in examining assumptions.

The Standard Model when born in the 1970s was thought to be good with only one Higgs boson all the way up to the grand unification scale at $10^{15} \mathrm{GeV}$. Since that time it has accumulated problems. It is hard to suppress strong CP violation. There is not enough CP violation to reproduce the baryon asymmetry of the universe. Longitudinal $W$ scattering is a problem if the Higgs mass is too high. There is no way to generate neutrino masses. It has 18 constants without a lepton sector CKM matrix and seven more with it. While the standard model has withstood all experimental tests, we know that it is only a low-energy (a few hundred GeV) approximation to a better model.

The most popular candidate to be the successor to the plain vanilla standard model is supersymmetry. It was introduced to stabilize the Higgs mass which is quadratically divergent in the standard model and only logarithmically divergent in the supersymmetric variants of the standard model. Supersymmetry does reduce to the standard model at "low" energies, but it also introduces 80 real and 44 complex constants. The theorists who are fans of supersymmetry are groping for variants that reduce these 124 new constants to a handful. If the supersymmetric successor to the standard model cannot reduce the total number of constants, it would seem to me to be a step backwards rather than an advance.

To the experimenters I would say that supersymmetry is a pure "social construct" with no supporting evidence despite many years of effort. It is okay to continue looking for supersymmetry as long as it doesn't seriously interfere with real work (top, Higgs, 
neutrinos, etc.).

String/brane theory is in a very different situation. It represents an attempt to bring together gravity and quantum mechanics, a problem worth serious effort. It has too many dimensions for those of us living in a four-dimensional world, but these are early days and perhaps they'll go away in the appropriate limit. There are several alternates that, on further examination, seem to turn into each other through duality transformations. Hidden in the center of all of these alternates are likely to be some kind of phase transitions that may lead to experimental signatures like those found for inflation. String/brane theory may even give the necessary constraints that supersymmetry needs to reduce the number of constants to a believable level. It is still too early to say, but it may be much more than metaphysics.

\section{Concluding Observations}

a) Experimenters (and phenomenologists) need to be more concerned about systematic errors and the tails on error-distribution functions.

b) Experimenters should learn more theory.

c) All theorists should have a required course in statistics before receiving their Ph.D.

d) We all hope for new things from LEP-II and the Tevetron, although the chances seem small. The new runs only increase LEP-II's mass reach by about $8 \mathrm{GeV}$ and the Tevetron's by $50 \mathrm{GeV}$.

e) We have big hopes for the $B$-Factories. The standard model's CP violation is not enough and new directions may become clear from the factories. The first results should be in next summer.

f) Neutrino physics is in ferment. More Super-K data, SNO, Borexino, KamLAND, Minos, K2K, should help to make things clear but it will take four to five years.

g) Some redundancy in neutrino experiments is useful; too much is wasteful.

h) I would love to see a low-energy muon-neutrino disappearance and reappearance experiment. Can it be done with Minos, or an appropriately designed CERNGran Sasso experiment?

i) LHC starts up in 2005 and we all hope to find out what is beyond our standard model. The experiments are huge and the sociology will be complex. Beware of too many boards and committees. 
j) An $e^{+} e^{-}$collider of 0.5 to $1 \mathrm{TeV}$ is a necessary companion to the LHC. It will only come to be if we all get behind it and push it as an international and regional program.

k) Muon colliders, VLHC's and exotic accelerator technologies are machines for 2020 or beyond. Muon colliders need R\&D work to demonstrate that damping can work and then we'll still face formidable problems. VLHC's are a fantasy now. In addition to cost breakthroughs they need some serious accelerator physics studies (for example, already at LHC some power supplies now need tolerances of one part in a million). Exotic techniques such as plasmas and lasers are still in their infancy. They have achieved accelerating gradients of $1 \mathrm{GeV}$ per meter, but only over a distance of a millimeter.

1) Muon storage rings as neutrino sources are interesting and much simpler than are muon colliders. A study of how to use polarization, mixed electron and muon neutrino beams and their antiparticles is needed. The machine and the experiments interact strongly.

m) Non-accelerator experiments in space and on the ground will be of increasing importance. The high-energy physics community should not be too parochial.

n) The string theorists are doing great things. I hope they justify or eliminate super symmetry and think up an experimental test.

o) There is an exciting future: the work will be difficult, expensive and rewarding. The young generation, with support from governments, can and will do it. This is not "The End of Science." 


\section{References}

[1] T. D. Lee and C. N. Yang, Phys. Rev. 104, 254 (1956)

[2] Bruno Pontecorvo, Inverse Beta Process, B. Pontecorvo, PD-205, Chalk River, Ontario, Canada, November 1946.

[3] Y. Suzuki, this conference.

[4] L. DiLella, this conference.

[5] LSND, Phys. Rev. C54, 2685 (1996).

[6] T. Mann, this conference.

[7] Fogli et al., hep-ph/9808205 and Phys. Rev. D59, 033001 (1999).

[8] Barger, et al., Phys. Rev. Lett. 82, 2640 (1999).

[9] Gonzales-Garcia, Phys. Rev. Lett. 82, 3202 (1999).

[10] Barger, Geer and Whisnont, hep-ph 9906487.

[11] P. Tsai, SLAC .

[12] J. Lykken, this conference.

[13] G. A. Voss, this conference.

[14] Ankenbrandt et al., Phys Rev ST-Accel. Beams 2 (1999), 081001.

[15] C. Contaldi, et al., Phys. Rev. Lett. 82 , 2034 (1999).

[16] S. Perlmutter, this conference.

[17] From M. Turner and J. Tyson, Rev. Mod. Phys. 71, S145 (1999). 